\title{
Gastric cancer after laparoscopic adjustable gastric banding: case report
}

\author{
Tomasz Szewczyk, Przemysław Janczak, Bogdan Modzelewski \\ Department of Gastroenterological, Oncological and General Surgery, Medical University of Lodz, Poland
}

Videosurgery Miniinv 2012; 7 (3): 210-212

DOI: 10.5114/wiitm.2011.27113

\begin{abstract}
Gastric cancer in patients who have undergone bariatric surgery is rare. The authors present a case of stomach cancer in a patient 6 years after adjustable silicone gastric band placement. The tumour was located below the band, not in its direct vicinity. Aetiological and risk factors of stomach cancer incidence in obese patients are discussed in this case study.
\end{abstract}

Key words: gastric cancer, gastric band, obesity.

\section{Introduction}

Stomach cancer in patients who have undergone bariatric surgery is rarely mentioned in the literature. The relation between the surgery and the neoplasm is equivocal and, with a very small number of reports, no decisive conclusions can be drawn.

\section{Case report}

The patient was 33 years old, with body mass index $(\mathrm{BMI})=46.69 \mathrm{~kg} / \mathrm{m}^{2}$ (height $175 \mathrm{~cm}$, weight $143 \mathrm{~kg}$ ). Gastroscopy was performed before the surgery. It did not reveal any changes within the oesophagus, stomach or duodenum. Presence of Helicobacter pylori was not determined. According to the patient's history, she had an adjustable silicone gastric band (Midband) placed laparoscopically on June $29^{\text {th }} 2005$. The band was well tolerated. The patient did not complain of vomiting or dysphagia. Regular weight loss was observed during the postoperative period. Follow-up appointments took place every 3 months in the first post-operative period and then every 6 months. By June 2008 the body mass had sta- bilized at 71-75 kg (BMI = 23.18-24.49 kg/m²). In October 2010, the patient contacted the hospital due to general feeling of illness, pain in the mesogastrium that increased after meals, and nausea. All the symptoms had lasted for a month. Panendoscopy was performed, which revealed the following. "In the middle of the body of the stomach, on the posterior wall, $2 \mathrm{~cm}$, conical hump visible with central, quite deep laceration (ulceration?) at the top, possible outside pressure. Samples were collected. No H. pylori was determined." The histopathology result was "adenocarcinoma non microcelullare male differentiatum". No infiltration of surrounding organs or distant metastasis were revealed. The patient asked to postpone the procedure until December. She had the operation on December $8^{\text {th }}$ 2010. Laparotomy was performed; the band was removed along with the larger portion of the greater omentum and visible, enlarged lymph nodes. The oesophagus was anastomosed end-to-back to a loop of jejunum with a mechanical suture and jejuno-jejunal Roux en $Y$ anastomosis was performed. Macroscopic assessment of the sample: the lesion was located in the 
middle of the stomach, in the area of the lesser curvature, on the anterior wall, with the diameter of about $2 \mathrm{~cm}$, with ulceration at the top. No complications were observed during the postoperative period. The patient was released on the $5^{\text {th }}$ postoperative day. The histological result of the excised stomach was as follows: "Adenocarcinoma male differentiatum ( $G$-3) partim microcellulare (Poorly cohesive carcinoma according to WHO 2010) corporis ventriculi cum infiltratione totalis parietis (pT4a). Type III tumour according to the Goseki classification, diffuse type according to Lauren. Macroscopically the lesion is sized $3 \mathrm{~cm} \times 2 \mathrm{~cm} \times 1 \mathrm{~cm}$. In the incision line, from the entrance to the stomach and duodenum, no neoplastic tissue was observed." 17 lymph nodes were found, all with reactive hyperplasia, without metastases. Immunohistochemical results are presented in Table I. The patient remains under the care of an oncologist.

\section{Discussion}

Obesity is conducive to the development of uterine, ovarian, breast, oesophageal and large intestinal cancer. The influence of increased BMI on stomach cancer incidence was assessed as questionable. Many reports indicate increased occurrence of the neoplasm in obese people. It especially concerns cases when the entrance of the stomach is affected in non-Asians [1]. There are numerous hypotheses indicating the biological mechanism of this phenomenon, starting from more frequent occurrence of gastro-esophageal reflux (GERD), insulin resistance, changes of leptin, adiponectin, GLP-1, glucocorticosteroids, sex hormones and proinflammatory factors levels [1-3]. The influence of leptin concentration on stomach cancer occurrence is an interesting hypothesis. It has been observed that an increased level of leptin increases the proliferation of stomach cancer cells in vitro. There are no studies assessing the level of leptin in patients with stomach cancer at early stages. Singular reports show only considerably lower concentration of leptin in patients with neoplasm [4]. It has been observed that concentration of leptin, considerably higher in obese patients, significantly decreases a few months after gastric band placement, which is correlated with the weight loss [5]. It should constitute a factor protecting against cancer. This observation is in agreement with reports concerning decreased stomach cancer incidence and mortality among patients after bariatric surgery $[6,7]$.
Table I. Immunohistochemical reactions in neoplastic cells

\begin{tabular}{|lc|}
\hline Keratin 7 & + \\
\hline Vimentin & + \\
\hline Smooth muscle actin & - \\
\hline TTF-1 & - \\
\hline CD117 & + \\
\hline Ki-67 index & $40 \%$ \\
\hline
\end{tabular}

The described risk factors of neoplasm occurrence following bariatric surgery are female sex, time after surgery (the longer, the higher risk), it is related to surgery excluding the stomach, with gastro-jejunal anastomosis performed, $H$. pylori, inflammatory reactions of the mucous membrane with dysplasia, and long-lasting exposure to deposited bile. Patients after restrictive surgery constitute a specific group. Such a procedure causes ongoing deposition of food which is conducive to action of local carcinogenic food factors. The effect of the increased pressure in the lumen of the alimentary tract with concurrent chronic mucositis and the influence of mechanical pressure caused by the band are also interesting $[8,9]$. Cancer incidence in the "excluded" stomach after Roux en $Y$ procedure is a particular diagnostic issue. Only indirect symptoms, usually uncharacteristic, such as persistent epigastric pain, nausea, diarrhoea, loss of appetite, or tumour palpable through the abdominal wall, suggest the diagnosis. Unequivocal diagnosis is only possible after computed tomography (CT) of the abdominal cavity, confirmed intraoperatively, has been performed. In the available literature, no cases similar to the presented one was found. Cancer occurrence after band placement was described, but it appeared above the band [10] or in the area where it was applied, and it was connected with its migration [9]. Risk factors which might have influenced the development of cancer in our patient are female sex and obesity lasting since childhood. Cancer occurred just 6 months after the procedure and was not located in its direct vicinity. It affected part of the stomach below the band. No H. pylori or stomach mucositis was observed. We assume the cancer had no relation whatsoever with the performed surgery and was merely a coincidence. Shortlasting ailments, small size of the tumour, and no distant metastases in the lymph nodes allow for 
a positive prognosis. Interestingly, the mother of the patient (55) is also under our care due to adjustable silicone gastric band placement in 2006. Body mass index $=47.97 \mathrm{~kg} / \mathrm{m}^{2}$ was an indication for the surgery. Both in gastroscopy before the procedure and in the examination performed in November 2010 no lesions were observed in the mother's stomach.

\section{References}

1. Yang P, Zhou Y, Chen B, et al. Overweight, obesity and gastric cancer risk: results from a meta-analysis of cohort studies. Eur J Cancer 2009; 45: 2867-73.

2. Adams TD, Hunt SC. Cancer and obesity: effect of bariatric surgery. World J Surg 2009; 33: 2028-33.

3. Sakra L, Siller J, Vyhnalek P, et al. Surgical treatment of gastric and small bowel gastrointestinal stromal tumours. Videosurgery Miniinv 2011; 6: 138-43.

4. Howard JM, Pidgeon GP, Reynolds JV. Leptin and gastro-intestinal malignancies. Obes Rev 2010; 11: 863-74.

5. Szewczyk T, Janczak P, Wyka K, et al. Influence of laparoscopic adjustable gastric banding on concentration of ghrelin and leptin. Clin Exp Med Lett 2009; 50: 187-91.

6. Adams TD, Stroup AM, Gress RE, et al. Cancer incidence and mortality after gastric bypass surgery. Obesity (Silver Spring) 2009; 17: 796-802

7. Sjöström L, Narbro K, Sjöström CD, et al; Swedish Obese Subjects Study. Effects of bariatric surgery on mortality in Swedish obese subjects. N Engl J Med 2007; 357: 741-52.

8. De Roover, Detry O, Desaive C, et al. Risk of upper gastrointestinal cancer after bariatric operations. Obes Surg 2006; 16: 1656-61.

9. Hackert T, Dietz M, Tjaden C, et al. Band erosion with gastric cancer. Obes Surg 2004; 14: 559-61.

10. Stroh C, Hohmann U, Urban H, Manger T. Gastric cancer after laparoscopic adjustable gastric banding. Obes Surg 2008; 18: 1200-2. 